\title{
Revisiting parallel catadioptric goniophotometers
}

\author{
Boris Karamata $^{* 1 a}$ and Marilyne Andersen ${ }^{\mathrm{a}}$ \\ ${ }^{a}$ Interdisciplinary Laboratory of Performance-Integrated Design (LIPID), LE Building - Station 18 \\ Ecole Polytechnique Fédérale de Lausanne (EPFL), 1015 - Lausanne, Switzerland
}

\begin{abstract}
A thorough knowledge of the angular distribution of light scattered by an illuminated surface under different angles is essential in numerous industrial and research applications. Traditionally, the angular distribution of a reflected or transmitted light flux as function of the illumination angle, described by the Bidirectional Scattering Distribution Function (BSDF), is measured with a point-by-point scanning goniophotometer yielding impractically long acquisition times. Significantly faster measurements can be achieved by a device capable of simultaneously imaging the far-field distribution of light scattered by a sample onto a two-dimensional sensor array. Such an angular-to-spatial mapping function can be realized with a parallel catadioptric mapping goniophotometer (CMG).

In this contribution, we formally establish the design requirement for a reliable CMG. Based on heuristic considerations we show that, to avoid degrading the angular-to-spatial function, the acceptance angle of the lens system inherent to a CMG must be smaller than $60^{\circ}$. By means of a parametric study, we investigate the practical design limitations of a CMG caused by the constraints imposed by the properties of a real lens system. Our study reveals that the values of the key design parameters of a CMG fall within a relatively small range. This imposes the shape of the ellipsoidal reflector and drastically restricts the room for a design trade-off between the sample size and the angular resolution. We provide a quantitative analysis for the key parameters of a CMG for two relevant cases.
\end{abstract}

Keywords: Scatterometry, Bidirectionnal Scattering Distribution Function (BSDF), Goniophotometry, Parallel measurement, Imaging goniophotometer, Catadioptric design, Ellipsoidal mirror

\section{INTRODUCTION}

A thorough knowledge of the angular distribution of light scattered by an illuminated surface under a given angle is essential in a large variety of scientific and industrial applications. Firstly, it provides quantitative information on the microscopic structure [1] and on the appearance of the surface itself, for instance for mastering the look of commercial products [2] or for computer rendering simulations [3]. Secondly, it can be used to calculate the distribution of light in a space illuminated by a multitude of secondary sources created by light interactions, such as for instance, the evaluation of stray light in an optical device or natural lighting in a room [4].

The angular distribution of a reflected or transmitted light flux as a function of the illumination angle can be described by the Bidirectional Scattering Distribution Function (BSDF) [5], which can be measured with an instrument called goniophotometer. A traditional goniophotometer generally consists of a two-axis mechanical scanner moving a photodetector around the sample in small angular steps across the hemisperical space [6]. The major drawback of such a scanning goniophotometer is its impractically long acquistion time. Indeed, assuming a time interval of one second between each measurement, the acquisition of a BSDF with a resolution of $1^{\circ}$ across the hemispherical space would take nearly six hours for a single incidence angle. Many applications such as computer rendering [7] or daylighting simulations $[4,8]$ generally require the characterizations of BSDFs for a large variety of materials and for numerous incidence angles and wavelengths.

*boris.karamata@a3.epfl.ch; phone+41.21.693.08.86 
In practice, these applications require orders of magnitude faster BSDF measurements. Such drastically faster measurements can be achieved only by simultaneously measuring light flux in all scattered directions. This requires a device capable of imaging the far-field distribution of light scattered by a sample onto a relatively small two-dimensional sensor array. Such an angular-to-spatial mapping function can be realized with a so-called parallel goniophotometer.

A few research groups and companies have devised a parallel goniophotometer, generally for a specific use in the first instance or for commercialization in the second one. Based on their working principles, these goniophotometers can be classified into three main categories, namely (i) screen imaging, (ii) dioptric angular mapping, and (iii) catadioptric angular mapping. The first category of instruments typically consists of a hemispherical screen intercepting light scattered over the entire hemisphere [2]. Light backscattered by the screen is then imaged on a sensor via an optical system. Instrument of the second category rely on a complex custom lens assembly performing the angular-to-spatial mapping by means of an optical Fourier transform [12]. The third category of instruments exploits a large parabolic or ellipsoidal mirror redirecting light toward a lens system [8 - 11].

The last category of parallel goniophotomers, which we designate catadioptric mapping goniophotometer (CMG), bears some inherent advantages such as a high throughput and negligible stray light, compared to the devices based on screen imaging. From a practical standpoint, for either laboratory or industrial applications, it is appealing to be able to build a custom parallel goniophotometer with a readily available lens system and ellipsoidal reflector (if not too large). The design of a dioptric angular mapping goniophotometer requires a deep knowledge of lens design and the cost of manufacturing of such a device would be prohibitive for a single instrument.

We start by explaining the working principle of a CMG (2). Then we establish the design requirements for a CMG incorporating a real lens system (3). By means of a parametric study, we investigate the impact of these requirements on the room for a practical design and analyze the quantitative figures calculated for the key parameters of a CMG for two relevant cases (4). We end up with a conclusion (5).

\section{WORKING PRINCIPLE}

In a catadioptric mapping goniophotometer (CMG), the simultaneous measurement of the far-field angular intensity distribution of light scattered by a sample relies on the optimal combination of a large reflector (catopric part) with a lens system (dioptric part). The primary function of the reflector is to reshape the light scattered by the sample within a large solid angle into either a converging or a nearly collimated beam so that it can be collected by a conventional lens system and dispatched on a two-dimensional detector array. Moreover, the shape of the mirror, which can be either ellipsoidal or parabolic, provides a known angular distribution of the reflected light beam at the lens system. This makes possible the accurate angular-to-spatial mapping of a direction of scattering onto a position on the sensor.

Since the design of a CMG based on a parabolic reflector is relatively straightforward and does not need thorough investigation, we will focus exclusively on the designs relying on an ellipsoidal reflector. The working principle is explained here below.

In a CMG relying on an ellipsoidal design, the sample and the lens system are located at the first and second focal points $\left(F_{1}\right.$ and $\left.F_{2}\right)$, respectively. To get some insights into the angular-to-spatial mapping performed by a CMG, assume a very small sample relative to the ellipsoidal reflector $(\mathrm{d} \rightarrow 0)$, and an ideal lens system that behaves like a thin lens (TL), whose optical axis coincides with the main axis of the ellipsoid, positioned exactly at $F_{2}$ (see figure 1). Thanks to the geometrical properties of an ellipse, namely the stigmatic relationship between the two foci $F_{1}$ and $F_{2}$, any ray scattered by the small sample positioned at $F_{1}$ - independent of its direction - is directed towards $F_{2}$ after reflection on the ellipsoidal mirror (red dotted line in figure 1). After passing by the center of the thin lens, the ray continues its straight trajectory till reaching the detector (D) in the focal plane at a position defined by the vector $r=[\mathrm{Y}(\alpha) \mathrm{Z}(\beta)]$, where $\alpha$ and $\beta$ are the azimuthal and elevation angles of the reflected ray relative to the lens axis, repectively.

Therefore, under the above assumptions, there is a linear correspondence between the angles $\alpha$ and $\beta$ defining the trajectories of the reflected rays passing by $F_{2}$ and the coordinates $Y(\alpha)$ and $Z(\beta)$ measured on the detector. Since the relationship (non-linear) between a scattering direction $\operatorname{SD}(\theta, \varphi)$ and the direction of reflection $\operatorname{RD}(\alpha, \beta)$ can be calculated with geometrical rules, the relationship between the angular intensity distribution of light scattered by the sample $\mathrm{I}_{\mathrm{S}}(\theta, \varphi)$ and the corresponding spatial intensity distribution on the two-dimensional array $\mathrm{I}_{\mathrm{D}}(\mathrm{y}, \mathrm{z})$ can be known. 
Therefore, by knowing the angular-to-spatial mapping function performed by a $\mathrm{CMG}\left(\mathrm{I}_{\mathrm{S}}(\theta, \varphi) \rightarrow \mathrm{I}_{\mathrm{D}}(\mathrm{y}, \mathrm{z})\right)$, one can extrapolate the angular intensity distribution of the rays scattered by the sample at $\mathrm{F}_{1}$, i.e. the BSDF of the sample.

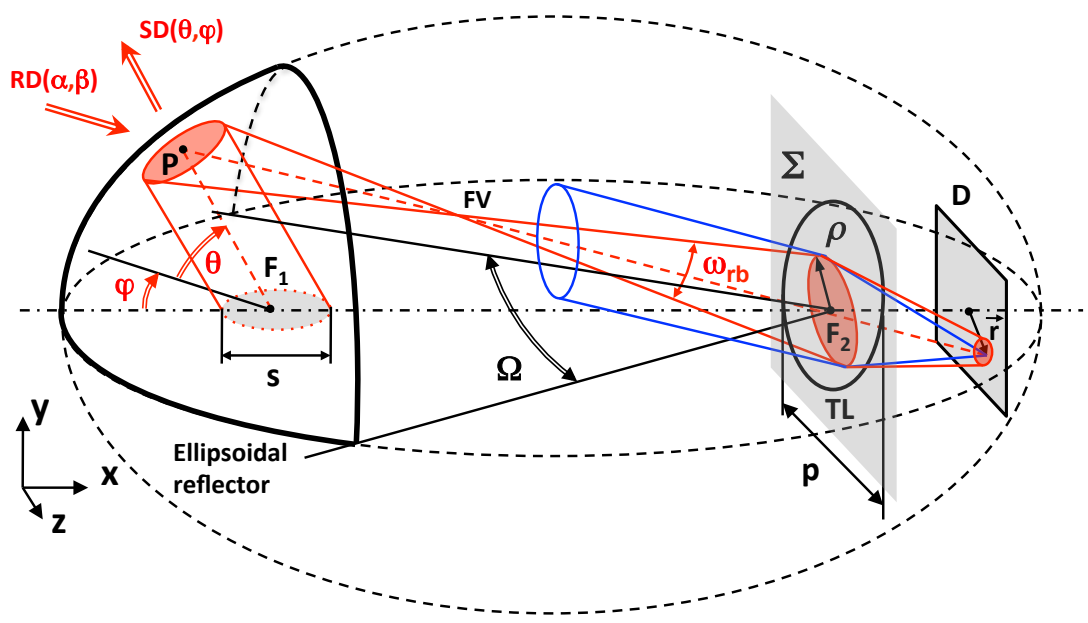

Figure 1. Working principle of a catadioptric mapping goniophotometer (CMG) with key components and parameters. Red lines: Path of a ray bundle scattered by the sample of finite size (diameter s) in a direction $\operatorname{SD}(\theta, \varphi)$ and its intersection with the plane $\Sigma$. The distance $\rho$ is the largest distance relative to $F_{2}$ and $p$ is the smallest entrance pupil diameter of the lens system to collect largest $\rho$ for all scattering directions. Red-dotted lines: Chief ray of the ray bundle. Blue lines: Path of a collimated ray bundle in the viewing direction $\operatorname{RD}(\alpha, \beta)$ focused by a thin lens (TL) and its intersection point $\mathrm{r}(\alpha)$ at the detector (D).

So far in our explanations we considered an infinitely small sample, i.e. a single ray (red dotted line) emitted at $F_{1}$ in a given direction. Let us now examine the more relevant case a collimated ray bundle emitted by a sample of finite size in a given direction $\operatorname{SD}(\theta, \varphi)$. As depicted in figure 1 (red lines), the ellipsoidal mirror makes the ray bundle converge into a focusing volume (FV) before it enters the thin lens (TL). The latter focuses the resulting diverging ray bundle on a spot roughly centered on a point of the detector defined by the vector $r$. For an ideal angular-to-spatial mapping, the ray bundle should be focused on a single spot. Under the rules of paraxial optics, this would be the case for a collimated ray bundle entering the thin lens as shown in blue lines in figure 1.

The largest focusing spot caused by the diverging ray bundle entering the lens simply accounts for a loss of angular resolution. The angular resolution is determined by the angle of divergence of the ray bundle, which is not rotationally symmetrical relative to its axis of propagation $\left(\mathrm{PF}_{2}\right)$. As shown in figure 1, the largest divergence angle for a focused ray bundle, corresponds to the angle $\omega_{\mathrm{rb}}$ defined in a longitudinal plane containing the axis $\mathrm{PF}_{2}$. Thus, the angle $\omega_{\mathrm{rb}}$ corresponds to the lowest angular resolution for a given scattering direction.

For our investigation need to know the lowest angular resolution, i.e. the largest focusing angle $\omega_{\mathrm{rb}}$, for all the directional ray bundles collected. We designate the largest of the focusing angles $\omega_{\mathrm{rb}}$ by $\omega$. The method used for the calculation of $\omega$ is provided in section 4.2 .

\section{DESIGN REQUIREMENTS}

\subsection{Requirements for an ideal lens system}

In this section we establish the requirements with which the lens system of a CMG must comply. As seen in 1.1, the reflector of a CMG reshapes the light scattered into a beam directed towards the lens system. The spatial and angular extents of the beam, which are determined by the design of the CMG and by the sample size, impose requirements to the lens system. In order to formally establish these requirements, we need to define the key parameters for describing the spatial and angular properties of the beam and of the lens system. Moreover, we will need to consider the inherent properties of a real lens system. 
The relevant dimensional parameters for the reflected beam are its largest angle of focusing ( $\Omega$ in figure 1$)$ and its largest size at the entrance of the lens system. In the first approximation, the angle $\Omega$ is mainly determined by the size and ellipticity of the reflective mirror (see 4.2). The largest beam size is defined by its radial distance $\rho_{\mathrm{m}}$ relative to $\mathrm{F}_{2}$ in the plane $\Sigma$. A radial distance $\rho$ (see figure 1) is defined for each ray bundle corresponding to a different direction of scattering. The distance $\rho_{\mathrm{m}}$ corresponds to the largest distance $\rho$ in the plane $\Sigma$. In section 4.2 , we provide the method used to calculate $\rho_{\mathrm{m}}$ as well as the intersections of a few ray bundles in the place $\Sigma$ calculated for a specific case. For simplifying the notations we define the distance $\mathrm{w}=2 \rho_{\mathrm{m}}$ as the smallest diameter centered on $\mathrm{F}_{2}$ encircling the full beam waist (see figure 3 ).

The relevant dimensional parameters for the lens system are its acceptance angle $(\Phi)$ and the effective size of the entrance lens allowing a light beam into the system without truncation. For the ideal case of a thin lens (TL), obviously, the effective lens aperture corresponds to the size of the lens itself. The latter also corresponds to the entrance pupil of the lens with a diameter $\mathrm{p}$ (see figure 1).

Obviously, since the whole beam must be collected, the lens system of a CMG must comply with the following two basic requirements:

(i) $\Phi>\Omega$

(ii) $\mathrm{p}>\mathrm{w}=2 \rho_{\mathrm{m}}$

Moreover, to be able to achieve the angular-to-spatial mapping function described in 1.1, the lens system of a CMG must comply with a third basic requirement:

(iii) The mid-point of the entrance pupil of the lens system is coincident with $\mathrm{F}_{2}$

As explained in the next section, the possibility to comply with the above specifications very much depends on the properties of the entrance pupil of the lens system.

\subsection{Properties of a real lens system and impact on a CMG}

So far we assumed a lens system with an entrance pupil with ideal properties, i.e. with a size and position independent of the angular field. Depending on the lens system, the entrance pupil generally varies in size, shape and orientation [13] as a function of the angular field $\alpha$ relative to the lens axis. The underlying reason for this dependence is to be found in the definition of the entrance pupil, which rests on the paraxial (also called first order, or gaussian) optics approximation that implies very low angles relative to the optical axis $\left(\alpha \rightarrow 0^{\circ}\right)$. Actually, the entrance pupil is the aperture stop as would be seen from a point on the optical axis. Generally, a different image (size, position and orientation) of the aperture stop is obtained at a large angular field.

Obviously, the properties of an angular dependent entrance pupil, designated here by $\operatorname{AP}(\alpha)$, are different than the ones of a paraxial pupil, whose two key properties are briefly recalled. First, independent of the angular field ( $\alpha)$, the prolonged central ray of a collimated ray bundle entering the lens system, called the principal or chief ray (CR), passes by a unique point, which corresponds to the mid-point of the entrance pupil. Second, the size of a collimated ray bundle entering a lens (d) system is determined by the size of the entrance pupil.

An angular dependent entrance pupil can severely impact the angular-to-spatial mapping function in a CMG as suggested in figure 2. This figure shows a lens system with an angular dependent pupil $\operatorname{AP}(\alpha)$ with very different properties at the two angular fields $\alpha_{1}$ and $\alpha_{2}$. The pupil $\mathrm{AP}_{1}$, which corresponds to a relatively low angular field $\alpha_{1}$, yields an entrance diameter $d_{1}$ and is located at the focal point $F_{2}$. The pupil $A_{2}$, which corresponds to a relatively large angular field $\alpha_{2}$, yields an entrance diameter $d_{2}$ - significantly smaller than $d_{1}$ - and is located at a point $\xi_{2}$ on the optical axis.

To emphasize the importance of the pupil properties we investigate qualitatively how a lens system with an angular dependent entrance pupil impacts the performance of CMG.

Firstly, since $\operatorname{AP}(\alpha)$ cannot be centered on $\mathrm{F}_{2}$ our third requirement cannot be fulfilled and the chief ray of an oblique ray bundle entering the lens system $\left(\mathrm{CR}_{2}\right)$ generally points towards a point $\left(\xi_{2}\right)$ which does not coincide with $\mathrm{F}_{2}$.

Since $\mathrm{CR}_{2}$ passes by $\xi_{2}$, the stigmatic relationship between $F_{1}$ and $F_{2}-$ on which the working principle rests (see 2) does not hold anymore. This has far reaching consequences: the ray $\mathrm{CR}_{2}$, which is the center of gravity of the ray bundle, originates from a point $\xi_{1}$ not coincident with $\mathrm{F}_{1}$. In other words, the position of the field of view depends on the position of the entrance pupil. This means that an angular dependent entrance pupil induces a spatial dependence in the measurement of intensity scattering distributions. 


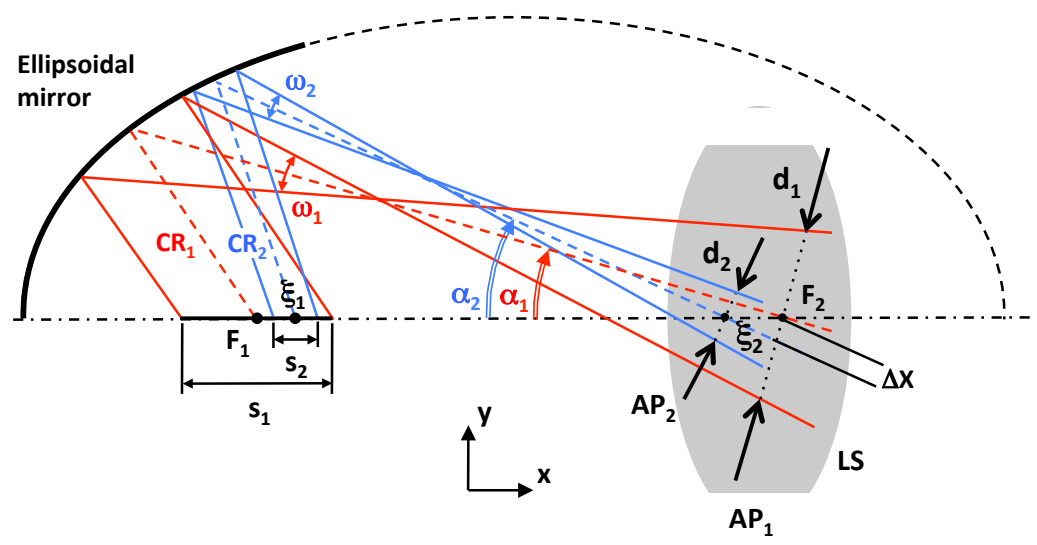

Figure 2. Impact of an aperture dependent entrance pupil $\operatorname{AP}(\alpha)$ on the angular-to-spatial mapping function in a CMG incorporating a real lens system (LS).

Consider now the effect of a change of pupil size. The size of the pupil $p(\alpha)$ determines the spatial extent of the ray bundle entering the lens system $(d(\alpha))$. The effective lens entrance size $d_{2}$ allows a smaller ray bundle into the lens system than $d_{1}$. Like in any optical system, this yields a loss of power throughput. More importantly, in a CMG, the size of the ray bundle is directly related to the sample size that can be measured. Figure 2 shows the largest sample size $s_{1}$ and $s_{2}$ that can be measured for the effective lens entrance sizes $d_{1}$ and $d_{2}$, respectively. In addition, as shown in figure 2, a ray bundle of smaller diameter results in a smaller focusing angle $\left(\omega_{2}<\omega_{1}\right)$, i.e. in a higher angular resolution (see 2$)$ in the plane xy. Therefore, in a CMG, the size of the entrance pupil of the lens system plays a crucial role regarding the largest measurable sample size and the angular resolution. An optimal trade-off must be found for each specific application.

Therefore, the angular-to-spatial mapping function of a CMG strongly relies on the properties of the entrance pupil of the lens system [14]. Despite the large variety of existing lens designs, there is a trend related to the acceptance angle of a lens system. Generally, for a lens system with acceptance angle lower than $60^{\circ}$, the lens designer strives to design for an a optical system with paraxial-like properties, i.e. the entering ray bundles are directed towards the same point. In practice, vignetting issues are not manageable for larger angular fields [15]. The design of a wide-angle lens, i.e. with an acceptance angle typically exceeding $90^{\circ}$ requires a radically different optimization procedure and trade-off to cancel out vignetting effects. Such multi-angle design optimization implies that the prolonged chief ray trajectories do not pass anymore by a single point for the whole angular field, which translates into grid distortion [15]. Moreover, such design results in a significant reduction of the size of the effective lens entrance (d). Between, $60^{\circ}$ and $90^{\circ}$ there is no clear design trend and lens systems with very different properties can be found.

We can conclude from our heuristic considerations that it would be perilous to use lenses with acceptance angles larger than $60^{\circ}$ in a $\mathrm{CMG}\left(\Phi<60^{\circ}\right)$.

To get a quantitative estimate of the constraint set by the size of the effective lens entrance (d) we have extrapolated the value of $\mathrm{d}$ for ten lenses taken from a database of lenses modeled in ZEMAX. We found out that $d$ lies in a range between $30 \mathrm{~mm}$ and $40 \mathrm{~mm}$. We will consider the middle range value, i.e. that $\mathrm{d}=35 \mathrm{~mm}$, for the upper limit of the effective entrance size of the lens. Our requirements are updated accordingly in the next section.

\subsection{Requirements for a CMG with a real lens system}

Taking into account our findings of the last section, the requirements for a CMG must be updated as follows:

(i) $\Phi<60^{\circ}>\Omega$

(ii) $\mathrm{d}<35 \mathrm{~mm}>\mathrm{w}=2 \rho_{\mathrm{m}}$

(iii) The mid-point of the entrance pupil of the lens system is coincident with $\mathrm{F}_{2}$ 
In the next section we will investigate the room left for a practical design of a CMG when complying with our consolidated design requirements.

\section{ROOM FOR THE DESIGN OF A CMG WITH A REAL LENS SYSTEM}

\subsection{Description of the parametric study}

A CMG with a good design must provide the angular resolution required, while ensuring an angular-to-spatial-mapping function free of artefacts. To avoid artefacts the CMG must incorporate a lens system that complies with our consolidated requirements (3.3).

Moreover, the design must meet practical requirements related to the finite sizes of a few key components. First, a CMG must incorporate a lens system, whose size is necessarily limited. Second, the size of the instrument, which is mainly determined by the size of the reflector, depends on the application. Typically, a portable instrument should be smaller than 10 centimeters while a laboratory instrument could be one order of magnitude larger. Finally, the design must provide a correct measurement for a given sample size, which varies a lot depending on the application.

To meet both these fundamental and practical requirements, the best trade-off should be found between the following parameters (described in sections 2 and 3.1): the sample diameter (s), the angular resolution $(\omega)$, the size of the ellipsoidal reflector (characterized by its semi-axis a), the reflector's ellipticity $(\varepsilon=\mathrm{F} / \mathrm{a}$ ), the beam waist (w) and the beam focusing angle $(\Omega)$.

Since these parameters are interrelated, a parametric study is best suited to get some insights into the room available for a practical design. The geometrical relationship between these parameters is shown in figure 1 and the calculation method is provided in the next section. In our study, we will investigate separately the case of a relatively small instrument $(\mathrm{a}=$ $100 \mathrm{~mm})$ and the case of a relatively large instrument $(\mathrm{a}=1000 \mathrm{~mm})$.

Our analysis of the parametric study will show that, in a practical design, the fundamental and practical design requirements constrain the values of the key design parameters to relatively small ranges drastically restricting the room for a trade-off.

\subsection{Method for calculation}

We need to calculate the following parameters defined in sections 2 and 3.1: the largest beam waist $\left(\mathrm{w}=2 \rho_{\mathrm{m}}\right)$, the largest beam focusing angle $(\Omega)$, and the lowest angular resolution $(\omega)$.

Light propagating in a given scattering direction $\operatorname{SD}(\theta, \varphi)$ corresponds to a bundle of parallel rays (Figure 1). For our investigation it is sufficient to model such a ray bundle with its peripheral rays. In all our simulations, a ray bundle is modeled with 25 rays evenly spaced at the periphery of a circular sample (i.e. one ray every $15^{\circ}$ of azimuth angle).

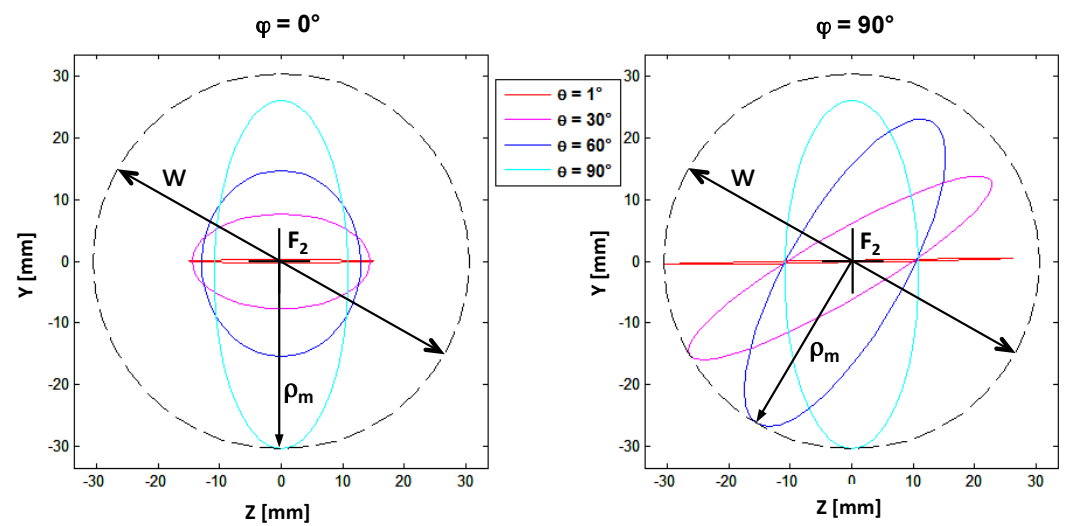

Figure 3. Results of ray tracing calculations with our Matlab code $(\varepsilon=0.6, a=600 \mathrm{~mm}, \mathrm{~s}=20)$. The intersection of reflected ray bundles with the plane $\Sigma$ (see figure 1) are shown for a few directions of scattering defined by the angles $\theta$ and $\varphi$. The radius $\rho_{\mathrm{m}}$, defined by the largest ray bundle intersection, determines the largest beam waist $(\mathrm{w})$ centered on $\mathrm{F}_{2}$ (that corresponds to the requirement of the smallest effective lens system size.) 
All the calculations described in this section are coded in Matlab. Figure 3 shows calculations results obtained for ray bundles intersecting the plane $\Sigma$ (see figure 1) for a few directions of scattering from the sample.

This calculation was performed for the following key parameters and values: sample diameter $\mathrm{s}=20 \mathrm{~mm}$, ellipse semiaxis a $=600 \mathrm{~mm}$, ellipticity $\varepsilon=0.6$. In this specific case, the entrance pupil must be approximately three times larger than the sample size (s) to allow collecting the largest ray bundle.

The direction $\operatorname{SD}(\theta, \varphi)$ of the ray bundle that defines $\mathrm{w}$ and $\omega$ is unknown a priori since it depends on the sample size relative to the ellipse size and on the ellipticity. Therefore we need to calculate the parameters $\rho$ and $\omega_{\mathrm{rb}}$ for a multitude of ray bundles scattered within a semi-hemispherical space ( $\pi$ steradian) and find out the largest figure for these two parameters. In all our simulations, the calculations of $\rho$ and $\omega_{\mathrm{rb}}$ are performed with 49 directions of ray bundles. This corresponds to a sampling every $15^{\circ}$ over $90^{\circ}$ for $\theta$ as well as for $\varphi$ thanks to the symmetry of the ellipsoidal reflector around its main axis.

The parameter $\omega_{\mathrm{rb}}$ is calculated as follows for a ray bundle. An angle of tilt relative to the beam axis P-F $\mathrm{F}_{2}$ is calculated for each of the 25 peripheral rays reflected on the ellipsoidal surface. For each pair of opposite peripheral rays, a focusing angle $\omega_{\mathrm{p}}$ is calculated by summing the tilt angles obtained. The focusing angle $\omega_{\mathrm{rb}}$ is defined as the largest of these peripheral focusing angles (see 2).

Note that, since the largest beam focusing angle $(\Omega)$ corresponds to the largest elevation angle considered, i.e. $\theta=90^{\circ}$ (see figure 3), our calculation must be made only for this angle. For a small sample relative to the ellipsoid $(\mathrm{d} \rightarrow 0)$, the angle $\Omega$ only depends on the ellipticity $(\varepsilon)$. The following expression for $\Omega$ was derived in [16]: $\Omega=2 \operatorname{atan}\left(\left(1-\varepsilon^{2}\right) / 2 \varepsilon\right)$.

\subsection{Results and analysis}

\section{$\underline{\text { Small CMG }}$}

Consider first the case of a relatively small instrument with an ellipsoidal reflector with a main semi-axis of dimension a $=100 \mathrm{~mm}$. The plot of figure 4 shows a calculation of the parameter $\Omega$ (blue curve) and of the parameter w (green curve) as a function of the reflector's ellipticity $(\varepsilon)$, for a sample size $\mathrm{s}=2 \mathrm{~mm}$. The parameter $\Omega$ decreases nearly linearly with $\varepsilon$, while the parameter $\mathrm{w}$ increases in an exponential-like manner with $\varepsilon$ above 0.35 . The slope of $w(\varepsilon)$ increases dramatically above $\varepsilon=0.8$.

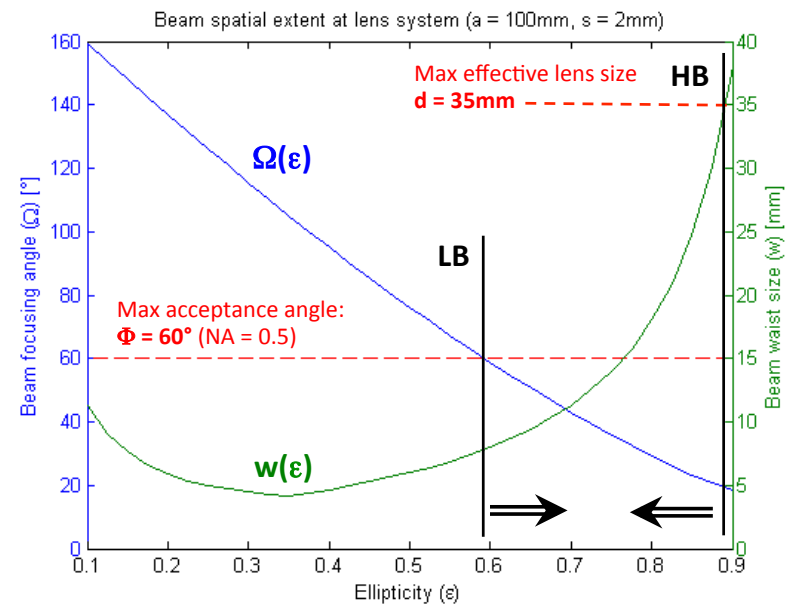

Figure 4. Results of calculation for $\Omega(\varepsilon)$ and $\mathrm{w}(\varepsilon)$ for a size of reflector a $=100 \mathrm{~mm}$ with a sample size $\mathrm{s}=2 \mathrm{~mm}$. Low-end design boundary set by the largest possible acceptance angle of the lens system (LB), and high-end design boundary set by the largest effective surface of the lens system (HB).

The practical limitations imposed by our design requirement for the lens system $\left(\Phi<60^{\circ}\right.$ and $\left.\mathrm{d}<35 \mathrm{~mm}\right)$ can be well appreciated thanks to this plot. To comply with the first requirement $\left(\Omega<\Phi=60^{\circ}\right)$, the ellipticity must be larger than approximately 0.58 , which sets the low-end design boundary $\varepsilon>0.58$ (LB). To ensure some margin of security and have 
more flexibility for choosing the lens system, the largest possible $\varepsilon$ is desirable. However, there is also a high-end design boundary (HB) that comes from the exponential-like growth of $\mathrm{w}$ with $\varepsilon$. To comply with the second requirement $(\mathrm{d}<35$ $\mathrm{mm}$ ), the ellipticity must be smaller than approximately 0.88 , which sets the high-end design boundary $\varepsilon<0.88$ (HB). Therefore, in the case of the relatively large CMG considered $(\mathrm{a}=100 \mathrm{~mm})$, the reflector's ellipticity lies within the range $\varepsilon=[0.65-0.85]$ (taking a margin of security with respect to the boundaries).

Let us investigate the practical design limitations on the angular resolution $(\omega)$ and on the sample size (s), implicitly imposed by the restrictions on $\varepsilon$. The parameters $s, \omega$ and $w$ are interrelated. The plot of figure $5 \mathrm{a}$ and $5 \mathrm{~b}$ show a calculation of $\omega$ (blue curve) and of $\mathrm{w}$ (green curve) as a function of $\mathrm{s}$, for the boundary values of the ellipticity $\varepsilon=0.65$ and $\varepsilon=0.85$, respectively. The practical design limitations on the sample size and on the angular resolution can be well appreciated thanks to this plot.

(a)



(b)

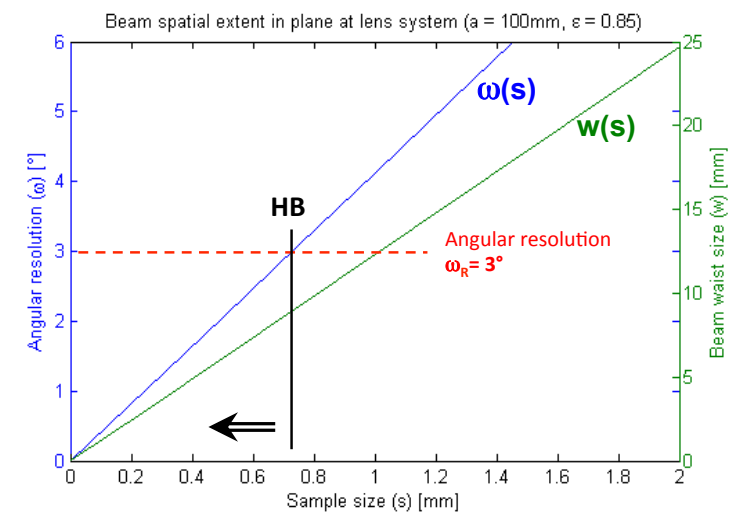

Figure 5. Results of calculation results for $\omega(\mathrm{s})$ and $\mathrm{w}(\mathrm{s})$ for a size of reflector a $=100 \mathrm{~mm}$ with a sample size $\mathrm{s}=2 \mathrm{~mm}$. (a) Ellipticity $\varepsilon=0.65$ and (b) $\varepsilon=0.85$. High-end design boundary set by the angular resolution required (HB).

The linear relationship between the sample size and the two parameters $\omega$ and $\mathrm{w}$ reveal that $\omega$ and $\mathrm{w}$ are proportional to $\mathrm{s}$. The beam waist $\mathrm{w}$ does not impose any further restriction on the sample size. However, with a sample of $2 \mathrm{~mm}$, the angular resolution is in the order of $6^{\circ}$, which is insufficient for most applications. An improvement of the resolution by a factor of two, i.e. the fulfillment of the requirement $\omega<\omega_{R}=3^{\circ}$, imposes a high-end boundary to the sample size (HB) whose impact on the sample size can be observed on the plots of figure 5 .

For the case $\varepsilon=0.65$ (figure $5 \mathrm{a}$ ) and $\varepsilon=0.85$ (figure $5 \mathrm{~b}$ ), the sample size must be smaller than approximately $0.75 \mathrm{~mm}$ and $1.5 \mathrm{~mm}$, respectively. A larger ellipticity allows measuring a larger sample.

Therefore, in a CMG of relatively small size $(\mathrm{a}=100 \mathrm{~mm})$ with $\varepsilon=0.75$, the room for a trade-off between the angular resolution and the sample size is determined by the expression $K_{S}=\omega / s$, where $K_{S}$ is a constant equal to two (3/1.5) and $\omega<\omega_{\mathrm{R}}$, the angular resolution required. A higher angular resolution can be obtained by proportionally reducing the sample size, and vice and versa. If there is no inferior limit to the sample size, the angular resolution of a CMG is ultimately limited by the angular resolution of the lens system.

\section{Large CMG}

Consider now the case of a relatively large instrument with an ellipsoidal reflector with a main semi-axis of dimension a $=1000 \mathrm{~mm}$. The plot of figure 6 shows a calculation of the parameter $\Omega$ (blue curve) and of the parameter $\mathrm{w}$ (green curve) as a function of the reflector's ellipticity $(\varepsilon)$, for a sample size $\mathrm{s}=10 \mathrm{~mm}$. Since, in the first approximation the parameter $\Omega$ is independent on the size of the reflector (see 5.2), the plot for the parameter $\Omega$ is identical to the one obtained for a CMG of smaller size (figure 4) and the low-end boundary (LB) is the same $(\varepsilon>0.58)$.

Due to the large size of the beam waist (w), which increases with the size of the CMG, the limited size of the optical systems $(\mathrm{d}<35 \mathrm{~mm})$ imposes severe limitations to the high-end boundary (HB) for $\varepsilon$. In the case investigated, the compliance with the requirement $\mathrm{d}<35 \mathrm{~mm}$, imposes that $\mathrm{HB}<\mathrm{LB}$, which is impossible. This means first that either a sample of smaller size must be considered or a lens system with larger $d$ must exist. We will make a marginal 
compromise on the sample size $(\mathrm{d}<10 \mathrm{~mm})$. Second, this means that, in the case of the relatively large CMG considered $(\mathrm{a}=1000 \mathrm{~mm})$, the reflector's ellipticity and the acceptance angle take the deterministic values $\varepsilon=0.6$ and $\Phi=60^{\circ}$, respectively.



Figure 6. Results of calculation for $\Omega(\varepsilon)$ and $\mathrm{w}(\varepsilon)$ for a size of reflector a $=1000 \mathrm{~mm}$ with a sample size $\mathrm{s}=10 \mathrm{~mm}$. Low-end design boundary set by the largest possible acceptance angle of the lens system (LB), and high-end design boundary set by the largest effective surface of the lens system (HB).

Let us investigate the practical design limitations on the angular resolution $(\omega)$ and on the sample size (s), implicitly imposed by the restrictions on $\varepsilon$. The plot of figure 7 shows a calculation of $\omega$ (blue curve) and of w (green curve) as a function of $\mathrm{s}$, for $\varepsilon=0.6$.

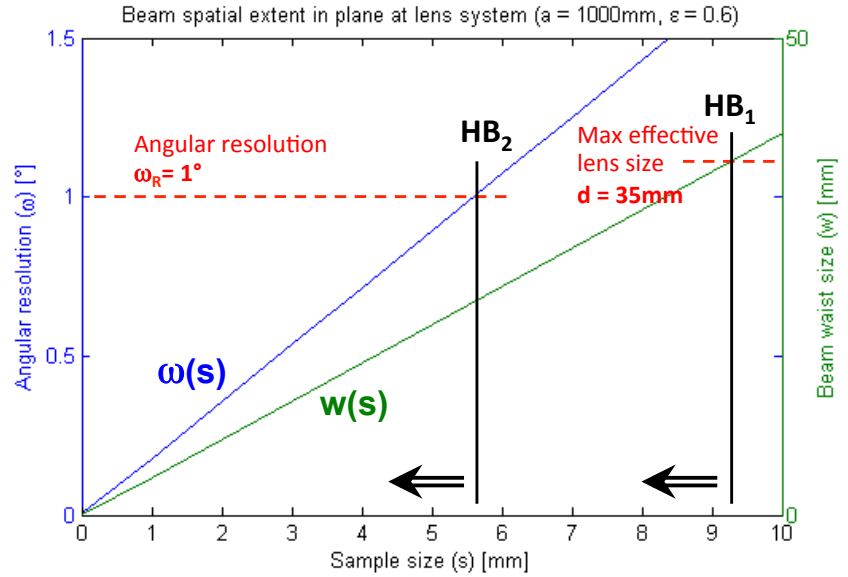

Figure 7. Results of calculation results for $\omega(\mathrm{s})$ and $w(\mathrm{~s})$ for a sample size $\mathrm{s}=10 \mathrm{~mm}$ with a reflector of size and ellipticity $\mathrm{a}=1000$ $\mathrm{mm}$ and $\varepsilon=0.6$, respectively. High-end design boundary set by the by the largest effective surface of the lens system $\left(\mathrm{HB}_{1}\right)$. Highend design boundary set by the angular resolution required $\left(\mathrm{HB}_{2}\right)$.

The practical design limitations on the sample size and on the angular resolution can be well appreciated thanks to this plot. Firstly, the plot $w(s)$ reveals a high-end design boundary $\left(\mathrm{HB}_{1}\right)$ due to the maximum effective size of the lens system $(\mathrm{d}<35 \mathrm{~mm})$. This boundary restricts the sample size to approximately $9.3 \mathrm{~mm}$ (trade-off on $\mathrm{s}$ necessary with $\varepsilon=$ 0.6 as mentioned above) and implies that the angular resolution will be better than around $1.1^{\circ}$. Secondly, the plot $\omega(\mathrm{s})$ reveals that the room for a trade-off between the angular resolution and the sample size is determined by the expression $\mathrm{K}_{\mathrm{L}}=\omega / \mathrm{s}$, where $\mathrm{K}_{\mathrm{L}}$ is a constant equal to approximately $0.12(1.1 / 9.3)$ and $\omega<1.1^{\circ}$. 
The requirement for a higher angular resolution $\left(\omega_{R}\right)$ than $1.1^{\circ}$ imposes a more restrictive high-end boundary $\left(\mathrm{HB}_{2}\right)$ to the sample size. As shown on figure 7, an angular resolution of $\omega_{\mathrm{R}}=1^{\circ}$ yields a maximum sample size $\mathrm{s}=5.6 \mathrm{~mm}$. If there is no inferior limit to the sample size, the angular resolution of a CMG is ultimately limited by the angular resolution of the lens system.

\section{CONCLUSION}

Our investigation reveals the practical limitations in the design of a CMG due to the constraints imposed by the properties of a real lens system. In particular, our parametric study shows that the fundamental and practical design requirements constrain the values of the key design parameters to relatively small ranges drastically restricting the room for a trade-off.

We found out that, for a CMG of relatively small size (reflector semi-axis of $10 \mathrm{~cm}$ ), the reflector's ellipticity must lie in a range between 0.65 and 0.85 and that the larger value allows for the largest sample size. The latter is in the range of 1.5 $\mathrm{mm}$ and yields an angular resolution of $3^{\circ}$. A higher angular resolution can be obtained by proportionally reducing the sample size, and vice and versa. To reach a higher resolution while keeping a relatively large sample size, a larger CMG is necessary.

Our investigation revealed that there is an ultimate limit to the size of a CMG that is in the order of one meter for the semi-axis of the ellipsoidal reflector. Moreover, the shape of the CMG, i.e. its ellipticity, is fully determined by the properties of the lens system. The value found for the ellipticity is $\varepsilon=0.6$. The largest possible sample size that can be used in such a CMG is of $9 \mathrm{~mm}$, which yields an angular resolution of around $1^{\circ}$. Like in the smaller CMG, the angular resolution improves by reducing the sample size (and vice and versa). However, it is challenging to fabricate an accurate enough ellipsoidal reflector of such a large size without impacting the angular resolution.

The relatively limited sample size that can be measured in a CMG is insufficient for applications that require the characterization of the average scattering properties of large objects such a as complex fenestration systems.

It must be remembered that our study rests on assumptions made on the properties of the lens system. Ideally, the design of a CMG should account for the design of the real lens system used and be performed with the help of a dedicated raytracing software. Unfortunately, the details of lens system are usually proprietary and hence not available in practice. Hopefully, our study provides a deeper understanding of the fundamental and practical limitations of a CMG that will provide helpful guidelines for the realization of future instruments.

\section{REFERENCES}

[1] Stover, C. J., [Optical Scattering: Measurement and Analysis], Robert E. Fisher and Waren J. Smith, Series Editors, (1990).

[2] Sipke, W. and Baumer, S., "Appearance characterization by a scatterometer employing a hemispherical screen," Proc. SPIE 5189, 163 - 173 (2003).

[3] Ward, G., "Measuring and modeling anisotropic reflection," Computer Graphics 26, 265-272 (1992).

[4] Ward, L.G. and Shakespeare R., [Rendering with Radiance: The Art and Science of Lighting Visualization], San Francisco: Morgan Kaufmann Publishers, (1997).

[5] Commission Internationale de l'Eclairage. Radiometric characteristics of material and their measurements. CIE 38 (TC-2.3), (1977).

[6] White, D. R., Saunders, P., Bonsey, S.J., van de Ven, J., and Edgar, H., "Reflectometer for measuring the bidirectional reflectance of rough surfaces," Applied Optics 37, 3450 - 3454 (1998).

[7] Dana, K. J., van Ginneken B., Nayar S., K. and Koendrik J., J., "Reflectance and texture of the real world surface," ACM Trans. Graph. 18, 1 - 34 (1999).

[8] Andersen M., E. Stokes E., Gayeski, N. and Browne, C., "Using digital imaging to assess spectral solar-optical properties of complex fenestration materials: A new approach in video-goniophotometry", Solar Energy, 84 549562, (2009).

[9] Mattison, P., Dombrowski, M., Lorenz, J., Davis, K., Mann, H., Johnson P. and Foos, B.,"The Hand-Held Directional Reflectometer: An angular imaging device to measure BRDF and HDR in real-time," Proc. SPIE 3426, $240-251$ (1998). 
Proceedings of the Optical Measurement Systems for Industrial Inspection VIII conference, SPIE Optical Metrology 2013, Munich, Germany, May 13-16, 2013

[10] Ren, J. and Zhao, J., "Measurement of a bidirectional reflectance distribution and system achievement based on a hemi-parabolic mirror," Optics letters 35(9), 1458 - 1460 (2010).

[11] Hahlweg, C. and Rothe, H., "Non-goniometric scatterometry - A review," Proc. SPIE 6682, D6620 - D6620 (2003).

[12] Boher, P., Luet, M., and Leroux, T., "Light scattered measurements using Fourier optics: A new tool for surface characterization," Proc. SPIE 5457, 344 - 354 (2004).

[13] Hughes, C., Denny, P., Jones E., and Galvin, M., “Accuracy of fish-eye lens models,” Applied Optics 49(17), 3338 - 3347 (2010).

[14] Laikin, M., [Lens Design], $4^{\text {th }}$ edition, Taylor and Francis Group, CRC Press, (2007).

[15] Smith's, W., J., [Modern Optical Engineering], $3^{\text {rd }}$ edition, MacGraw-Hill, 154 (2000).

[16] Rosete-Aguilar, M., Rodriguez-Herrera, O. G. and Bruce N., "Optical design of a scatterometer with an ellipsoidal mirror," Opt. Eng. 42(6), 1772 - 1777, (2003). 